\title{
DETAB: Modelo Colaborativo De Detecção E Alerta De Buracos Em Vias Pavimentadas
}

\author{
Thiago Roberto Lima Lopes ${ }^{1}$, Rodrigo da Rosa Righi ${ }^{1}$, Lucas Pfeiffer Salomão Dias ${ }^{1}$, \\ Cristiano André da Costa ${ }^{1}$ \\ ${ }^{1}$ Programa de Pos-Graduação em Computação Aplicada (PPGCA) \\ da Universidade do Vale do Rio dos Sinos (UNISINOS) \\ Av. Unisinos, 950 - Bairro Cristo Rei - São Leopoldo, RS, Brazil \\ \{lolthiago,lucaspfsd\}@gmail.com, \{cac,rrrighi\}@unisinos.br
}

\begin{abstract}
This article presents the Modelo Colaborativo de Detecção e Alerta de Buracos em Vias Pavimentadas (DETAB), which provides a distributed framework that automatically collects through ultrasonic sensors installed in the vehicle, analyzes and shares pothole data among drivers without any kind of human interaction. The applied technologies: HC-SR04 sensors, NodeMCU microcontroller, and an Android App target to be a lower cost solution with high accuracy. The results of both simulated (a toy and in-home lanes) and real (a car in a particular road) scenarios shows that the DETAB was able to map potholes and share the information with other drivers in a satisfactory fashion.
\end{abstract}

Resumo. Este artigo apresenta um modelo denominado Modelo Colaborativo de Detecção e Alerta de Buracos em Vias Pavimentadas (DETAB) o qual automaticamente detecta a existência de buracos, através de sensores ultrassônicos instalados no veículo, analisa e compartilha as informações relativas ao buraco com outros motoristas sem a necessidade de nenhum tipo de intervenção humana. As tecnologias envolvidas: sensor HC-SR04, micro controladora NodeMCU e um aplicativo para Android tem por objetivo ser uma solução de baixo custo, mas com alta acurácia. Os resultados obtidos através da avaliação do modelo em ambiente simulado, bem como em ambiente real, mostram que o DETAB foi capaz de identificar buracos na pista e também realizar o compartilhamento dessa informação com outros motoristas de forma satisfatória.

\section{Introdução}

Historicamente, o poder público não consegue fornecer uma ampliação da malha rodoviária asfaltada e uma manutenção efetiva que garanta qualidade duradoura, portanto, não acompanhando o aumento significativo no número de veículos ocorrido nas últimas décadas. Com o incremento de veículos sobre a mesma estrutura rodoviária, há o surgimento de problemas, sendo as péssimas condições das estradas um potencializador, em especial nos congestionamentos de tráfego e acidentes que findam em perda de vidas humanas. Estes fatos, além de notórios, também se encontram relatados pela Confederação Nacional do Transporte [CNT 2018].

A CNT no seu anuário do Transporte de 2016, mostra uma avaliação de $100.763 \mathrm{~km}$ de vias brasileiras asfaltadas e conclui que 57,2\% estão em condições gerais de regular a péssimo. Este dado torna-se mais impactante quando olha-se para alguns 
itens específicos como geometria, onde 77,2\% estão de regular a péssimo [CNT 2016]. Novamente a CNT, em 2018 [CNT 2018] mostra dados mais pungentes, onde 61,8\% das rodovias pesquisadas estão em condições gerais de regular a péssimo.

Buracos danificam rodas, suspensão, desalinham a direção, colaboram para o aparecimento de ruídos e prejudicam motoristas e passageiros. Eles são responsáveis por milhões de reais em manutenção veicular e execução de apólices de seguro. Na questão de prejuízos, conforme for o órgão responsável pela manutenção da rodovia o estado, o município ou a união podem ser responsabilizados, gerando mais despesas para o setor público. Desta forma, é de muita relevância identificar a localização dos buracos para tomada prévia de ações a fim de evitar acidentes e planejar melhor a rota de tráfego.

Um dos grandes problemas no planejamento da manutenção da rodovia é a falta de confiabilidade das medições manuais, o que pode levar a uma má priorização do trabalho de reparação. Alguns dos benefícios de uma coleta automatizada são a precisão e padronização dos levantamentos das condições e a capacidade de ser ampliado para atuar em qualquer âmbito, ajudando na redução de custos de manutenção em vários níveis.

Visando colaborar com a identificação de buracos, minimizando o risco à vida, este trabalho propõe o Modelo Colaborativo de Detecção e Alerta de Buracos em Vias Pavimentadas (DETAB). Diferentemente dos trabalhos relacionados, o DETAB combina módulos de hardware, computação móvel e integra frameworks para detectar e compartilhar informações sobre buracos com outros motoristas que trafegam próximos aos buracos existentes sem a necessidade de nenhuma intervenção humana.

O trabalho está organizado da seguinte maneira: na Seção 2 são apresentados os trabalhos relacionados; o modelo proposto e sua arquitetura são descritos em detalhes na Seção 3; as decisões tomadas e a tecnologia utilizada nesta implementação do modelo, são apresentados na Seção 4; a Seções 5 abrange a metodologia de avaliação, descrição dos cenários de testes realizados e os resultados obtidos, e; por fim, na Seção 6 são apresentadas as considerações finais e as expectativas de trabalhos futuros.

\section{Trabalhos Relacionados}

Foram analisados trabalhos relacionados que são o estado da arte e que se propõem a resolver problemas similares. Também fora elaborado uma tabela comparativa (Tabela 1), organizada de forma sequencial, mostrando as principais características.

1 - [Nienaber et al. 2015] compararam em seu trabalho três abordagens para o cálculo de problema de percepção de profundidade, que é encontrado durante a análise de buracos através de imagem. As abordagens foram: semelhança geométrica de triângulos, razão anarmônica e calibração da matriz de projeção da câmera.

2 - [Moazzam et al. 2013] apresenta uma alternativa para a análise tridimensional de imagens de problemas em pavimentação usando Microsoft Kinect Sensor. Uma limitação apontada é justamente vinculada ao hardware do Kinect que possui alcance de 4 metros, de acordo com dados oficiais do fabricante, e 3.5 metros de acordo com este trabalho.

3 - [Rishiwal and Khan 2016] apresentam um aplicativo para Android que realiza a detecção de buracos no pavimento através da vibração e aceleração do dispositivo móvel. Este trabalho apresenta uma taxa de $93.75 \%$ de exatidão na detecção de buracos. 
4 - [Vupparaboina et al. 2015] apresentam uma abordagem usando uma câmera e um laser fazendo com que seja possível de detectar buracos, secos ou com água, em vias secundárias. A prova de conceito apresentada demonstra uma taxa de assertividade de 98\% para buracos secos e $94.7 \%$ para buracos com água.

5 - [Madli et al. 2015] propõem um mecanismos de detecção de buracos atraves de sensor ultrassônico, que efetua alerta ao usuário através de um aplicativo para smartphone.

6 - [Murthy and Varaprasad 2014] apresentam uma abordagem baseada em visão 2D com tratamento de imagem feitos em frames capturadas de uma câmera do tipo pinhole. Este trabalho posso limitações relacionados as condições de luminosidade.

7 - [Klein and Borges 2016] criaram um aplicativo colaborativo para smartphone chamado Buracos Monitor, onde os usuários podem compartilhar informações sobre buracos. Este aplicativo possui um repositório de informações global externo, em um website, que pode ser compartilhado com o poder público.

Table 1. Comparação de trabalhos relacionados

\begin{tabular}{|c|c|c|c|c|c|c|c|c|}
\hline \multicolumn{9}{|c|}{ Trabalhos } \\
\hline Características & 1 & 2 & 3 & 4 & 5 & 6 & 7 & DETAB \\
\hline Tipo de detecção & Vídeo & $\begin{array}{l}\text { Visão } \\
\text { 3D }\end{array}$ & $\begin{array}{l}\text { Vibração/ } \\
\text { aceleração }\end{array}$ & Visão 3D & Sonar & Vídeo & N/A & Flexível \\
\hline Dispositivo & GoPro & Kinect & Android & Laser/Câm. & Ultrassom & Câmera & N/A & Extensível \\
\hline $\begin{array}{l}\text { Tem notificação ao } \\
\text { motorista? }\end{array}$ & N/A & N/A & N/A & N/A & SMS & N/A & Aplicativo & Aplicativo \\
\hline Distância de alcance & $30 \mathrm{~m}$ & $3.5 \mathrm{~m}$ & N/A & $20 \mathrm{~cm}$ & $4 \mathrm{~m}$ & $3.5 \mathrm{~m}$ & N/A & Relativo ao add-on \\
\hline Velocidade & $40 \mathrm{~km} / \mathrm{h}$ & N/A & N/A & N/A & N/A & N/A & N/A & Relativo ao add-on \\
\hline $\begin{array}{l}\% \text { precisão - buraco } \\
\text { seco }\end{array}$ & $98 \%$ & N/A & $93.75 \%$ & $98 \%$ & N/A & N/A & N/A & Relativo ao add-on \\
\hline $\begin{array}{l}\% \text { precisão - buraco } \\
\text { com água }\end{array}$ & N/A & N/A & N/A & $94.7 \%$ & N/A & N/A & N/A & Relativo ao add-on \\
\hline $\begin{array}{l}\text { Detecta profundida / } \\
\text { severidade? }\end{array}$ & Não & Profund. & Severid. & Profundid. & Profundid. & Severid. & N/A & Relativo ao add-on \\
\hline É colaborativo? & Não & Não & M2M & Não & M2M & Não & $\mathrm{H} 2 \mathrm{M}$ & M2M \\
\hline Notifica motorista? & N/A & N/A & N/A & N/A & SMS & N/A & Som/Visual & Som/Visual \\
\hline $\begin{array}{l}\text { Acesso web para au- } \\
\text { toridades? }\end{array}$ & N/A & Não & Sim & Não & Não & Não & Sim & Sim \\
\hline Precisa de internet? & N/A & Não & Não & N/A & Não & Não & Sim & Sim \\
\hline $\begin{array}{l}\text { Salva dados local- } \\
\text { mente? }\end{array}$ & N/A & N/A & Sim & Não & Não & Não & Sim & Sim \\
\hline Multi-sensor? & Não & Não & Não & Não & Não & Não & Não & Sim \\
\hline
\end{tabular}

É fundamental ressaltar que três pontos importantes diferenciam o modelo proposto dos demais trabalhos relacionados são: a) a capacidade de emitir alertas aos usuários motoristas, de modo a dar-lhes ciência do que está por vir e viabilizar a tomada de medidas cabíveis com antecedência, eliminando ou ao menos reduzindo os riscos de acidentes e danos materiais possíveis de serem causados; b) o modelo tem por objetivo ser uma solução de comunicação M2M, ou seja, não necessitando de nenhuma intervenção humana para que todo o processo ocorra; c) a oferta de um portal Web para autoridades encarregadas da manutenção das vias públicas, de forma a auxilia-los na organização dos pontos prioritários e que necessitam maior atenção. 


\section{Modelo Colaborativo De Detecção E Alerta De Buracos Em Vias Pavimentadas - DETAB}

O modelo DETAB é independente da tecnologia de implementação, sendo possível implementá-lo das mais diversas formas e tecnologias, a Figura 1 ilustra o modelo.

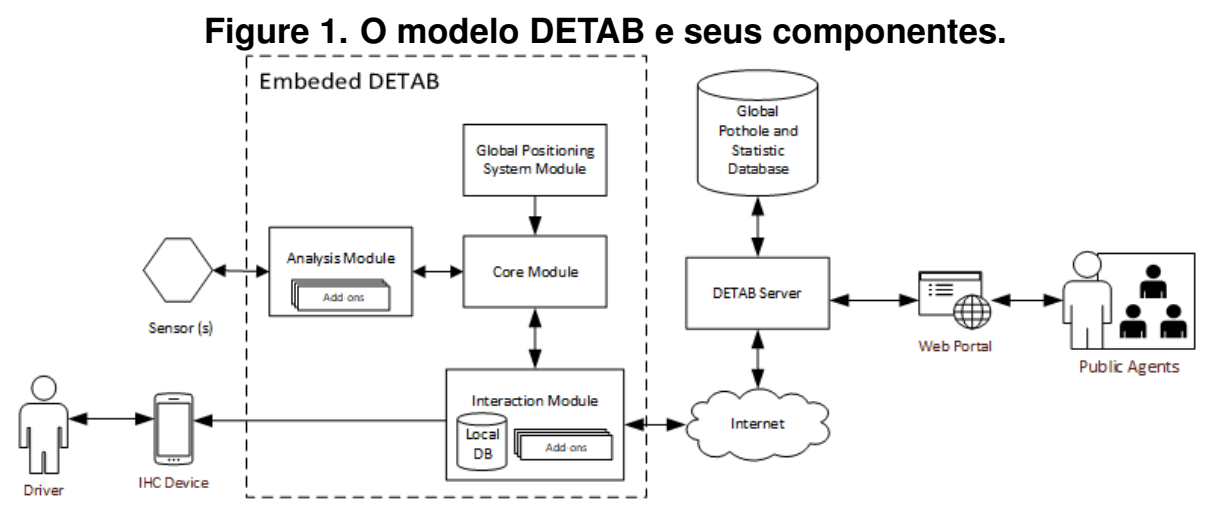

Conforme ilustrado na Figura 1 é possível visualizar a forma de interação no modelo, onde o DETAB trabalha junto aos sensores que são capazes de detectar irregularidades no asfalto. O DETAB ao detectar um buraco, calcula seu posicionamento geográfico (latitude e longitude) usando o sistema GPS e notifica o motorista sobre a(s) incidência(s) de buraco(s) à frente. O modelo proposto possuí algumas funcionalidades básicas que garantem sua melhor eficiência e são detalhadas abaixo:

1- Fornecer uma interface padrão para recepção de informações do módulo de detecção: esta funcionalidade garante com que o sistema possa trabalhar com diversos tipos de dispositivos e sensores como fonte de informação. Para tanto, são definidos quais dados são esperados e seus formatos, de modo que possam ser desenvolvidos add-ons normalizadores específicos para cada tecnologia que se deseja acoplar.

2- Notificar o usuário durante o trajeto: esta funcionalidade trata da apresentação para o usuário da informação sobre a existência de buraco(s) à frente. $O$ módulo que implementará esta funcionalidade terá por objetivo ser extensível à diversos dispositivos que tenham interfaceamento com o usuário, pois o foco principal é garantir a ciência do motorista sobre o(s) buraco(s) em seu percurso, independente da tecnologia adotada.

3- Enviar as informações coletadas para a base de dados global: esta funcionalidade garante que o modelo seja colaborativo, uma vez que, o modelo executando em um determinado veículo envie e receba informações da base de dados global, que por sua vez são oriundas do modelo executando em outros veículos.

\subsection{Arquitetura}

A arquitetura do DETAB é dividida em quatro módulos principais. Para que o modelo seja independente de tecnologia, emprega-se o conceito de $a d d$-on, tendo estes a responsabilidade de comunicação com uma determinada tecnologia, evitando que o modelo demande conhecimento qualificado de um nicho tecnológico. Este trabalho apresenta margem para três grandes grupos de add-ons, que também são apresentados a seguir. 
Add-ons de análise específica por tipo de entrada - Devem ser interpretar sinais produzidos por sensores, e notificar o módulo de análise, respeitando a interface de comunicação que determina um padrão de formato dos dados, quando um buraco for identificado.

Add-ons de interação com o usuário motorista - Devem interagirem com outros sistemas que fazem comunicação com o usuário motorista, convertendo a informação recebida em um determinado formato para o formato requerido pela aplicação.

Add-ons de persistência e colaboração entre partes do modelo - Devem salvar e compartilhar a informação sobre a existência de buraco em determinada posição geográfica. Este satisfaz a parte colaborativa uma vez que os dados compartilhados podem ser consumidos por veículos que também estejam executando o sistema.

Analysis Module - Este módulo tem por responsabilidade encapsular múltiplas implementações de algoritmos capazes de lidarem com diversos tipos de entrada de dados, que dependem basicamente do tipo de sensor utilizado. Para este módulo não encontrarse atrelado a um determinado tipo de sensor ou tecnologia, ele atua como um dispatcher, executando add-ons que realizam a analise especializada para cada tipo de sensor. Este módulo, após a detecção realizada por um ou mais add-ons, coleta as informações fornecidas e invoca o Core Module, para dar seguimento ao processo.

Core Module - Este modulo atua como orquestrador que interliga todos os demais módulos do sistema. Sua atuação inicia em forma de reação a uma notificação do Analysis Module. Ao ser notificado, obtendo as informações provenientes do(s) sensor(s) este módulo usa o módulo de posicionamento para obter suas coordenadas geográficas (latitude e longitude) de forma a compor uma informação completa. Estas informações serão redirecionadas para o módulo de interação para dar seguimento ao processo.

Interaction Module - Este módulo é responsável por fazer a interação com o meio externo através de lançamento de eventos, onde um evento trata-se da identificação de um buraco. Um dos objetivos que este módulo visa atender é a funcionalidade acima descrita de notificar o usuário durante o trajeto, bem como enviar as informações coletadas para a base de dados global. Para este módulo não atrelar-se a tecnologia ele também atua com um dispatcher, que faz a propagação de eventos para add-ons registrados. Neste contexto, a ação a ser tomada e os detalhes de como fazê-la, ao lançar-se um evento são definidos pelo add-ons que está registrado, que podem ser nenhum ou muitos.

Global Positioning System Module - Este módulo é responsável por fornecer ao módulo requisitante, neste caso o módulo principal, as informações de posicionamento geográfico (latitude e longitude). Estas informações podem ser obtidas através do dispositivo GPS (Sistema de posicionamento global) presente nos smartphones ou através da central multimédia em veículos que possuem um sistema GPS de fábrica.

Web Portal - O portal web visa ser uma forma fácil e eficaz de colaborar com o poder público, oferecendo uma ferramenta onde seja possível verificar-se a ocorrência de buracos e sua severidade de forma a guiar a priorização das ações realizadas para manutenção das vias públicas. Este portal web oferece mecanismos para os agentes do poder público assinalarem que determinado(s) buraco(s) foi(ram) concertado(s).

DETAB Server - O Detab Server visa prover uma interface de consulta e 
alimentação de dados online de forma a ser um $h u b$ dos sistema rodando nos veículos, bem como para o portal web. Este servidor também tem por responsabilidade filtrar e eliminar mapeamentos duplicados, ou seja, o mesmo buraco mapeado por outros motoristas, bem como encapsular o acesso aos bancos de dados.

\section{Decisões de Tecnologia e Implementação}

Conforme o modelo proposto, foi desenvolvido um protótipo que implementa: O sistema embarcado, o aplicativo de interação com o motorista, além do lado server-side. Este trabalho parte da premissa de que os sensores e os algoritmos empregados para análise e detecção de buracos já existam, então dentro das possibilidades, optou-se pelo sensor ultrassônico HC-SR04 utilizado em [Madli et al. 2015], conectado a uma placa controladora ESP8266-WiFi-SOC da Espressif com o firmware NodeMCU 1.0. Existem diferentes tipos de sensores ultrassônicos com diferentes faixas de transmissão e ângulos de detecção, todavia, o HC-SR04 se demonstrou em particular atrativo devido sua popularidade, baixo custo e estar presente em outros trabalhos relacionados.

O add-on de interação com o motorista mostra os alertas de buracos através de um aplicativo Android. Neste aplicativo também foram imputadas as responsabilidades do Core Module, Global Positioning System Module e Interaction Module. O dispositivo utilizado nos testes de avaliação foi o Motorola Moto X Style, com Android versão 6.0, 3GB de memória RAM e processadores: Dual-core 1.8GHz + Quad-core 1.44GHz. Este aparelho tem uma precisão de GPS que varia de 3 à 5 metros e utiliza os sistemas A-GPS e GLONASS combinados para aumentar a precisão. Para a implementação do Detab Server foi escolhido a tecnologia de ASPNET Web API, de modo a fornecer dados ao aplicativo, questões de autenticação e inserção de novos buracos mapeados.

\section{Metodologia de Avaliação: Experimentos e Resultados Obtidos}

A elaboração de uma forma de análise do sistema com viés quantitativo resultou na criação de uma linha de avaliação que contemple testes de funcionalidade e de integração do sistema, mais especificamente nas questões de: A) detecção de buracos por parte do sensor; B) envio dessas informações para o aplicativo Android; C) pela emissão de alerta pelo aplicativo para o motorista, e por fim, a submissão dos buracos ao DETAB Server.

\subsection{Cenário: Teste com ambiente e buracos simulados}

A fim de formar um método de teste exequível repetitivamente, criou-se um cenário de pequena escala onde foi fixado na parte frontal de um carro de controle remoto (que tem $15 \mathrm{~cm}$ de altura), o sensor HC-SR04, apontando para baixo, portanto, formando ângulo de 90 graus em relação ao solo. Este sensor ficou disposto a uma altura de $12 \mathrm{~cm}$ em relação ao solo, sendo o sistema pré-configurado para uma tolerância de $2 \mathrm{~cm}$, ficando assim, definido como buraco valores lidos que excedam o limite de $15 \mathrm{~cm}$ de altura.

Para simular o ambiente foi desenvolvido um percurso usando 4 folhas de isopor de $1 \mathrm{~m} \times 33 \mathrm{~cm} \times 20 \mathrm{~mm}$, totalizando 4 metros de pista, onde foram dispostos 5 buracos de aproximadamente $13 \mathrm{~cm} \times 8 \mathrm{~cm}$ em espaçamento aleatório. Nestas folhas de isopor também foram inseridas cavidades que não são considerados buracos devido sua profundidade ser inferior a tolerância, de modo a testarmos se este o sistema não iriam emitir falso positivo. A Figura 2 é uma montagem de fotos mostrando a criação da pista de teste usando isopor, bem como a instalação e fixação do sensor no carro de controle remoto. 
Figure 2. Fotos tiradas durante os testes do cenário simulado.

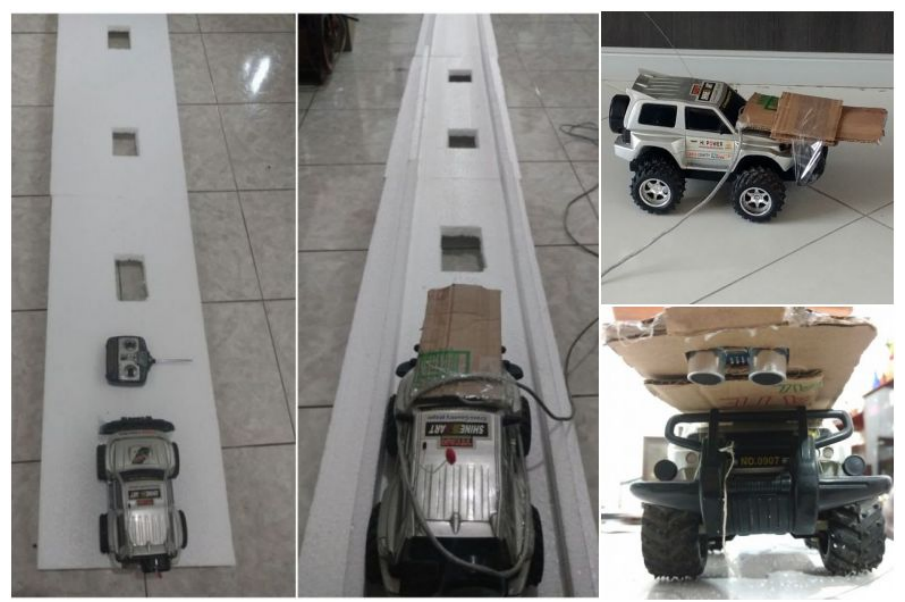

Figure 3. Buracos mapeados durante o teste em ambiente simulado.

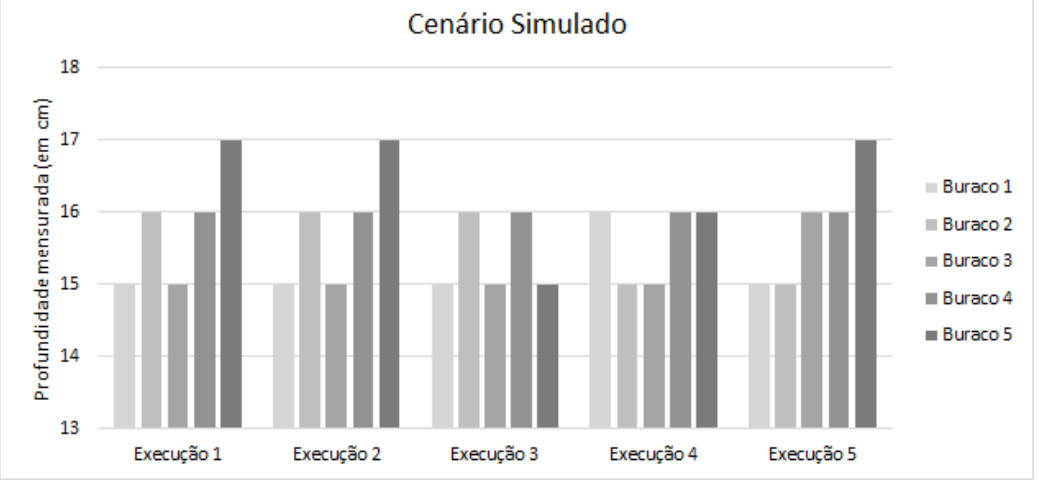

Assim, o experimento proposto se baseou em realizar a simulação em uma bateria de 5 execuções, e com os resultados coletados em cada uma delas efetuar um comparativo com a quantidade efetiva de buracos, além de verificar se houve a notificação de mapeamento pelo aplicativo.

O gráfico apresentado na Figura 3 apresenta as profundidades mensuradas para cada um dos cinco buracos em cada uma das cinco execuções. Evidencia-se que todos os buracos foram mapeados corretamente e notificados ao aplicativo Android, apenas considerando-se uma variação entre as medidas atribuída à precisão do sensor utilizado.

\subsection{Cenário: teste em ambiente real}

De modo a levar o experimento a um nível mais concreto, instalou-se o sensor HC-SR04 em um veículo de passeio, VW Voyage, cujo qual tem um altura padrão em relação ao solo de $16 \mathrm{~cm}$. O sensor foi disposto da mesma forma dos testes realizados no ambiente simulado, ou seja, instalado na parte frontal, apontando para baixo. Todavia, neste cenário o sensor ficou disposto a uma altura de $30 \mathrm{~cm}$ do solo. A placa controladora foi instalada no interior do veículo e alimentada através de uma tomada de 12 volts do próprio carro.

Para execução dos testes foi escolhido a rua Manoel Osório Ourique, Girassol, Gravataí/RS, dadas suas características: ser asfaltada, reta, plana e possuir buracos de variados formatos, tamanhos e profundidades. O cenário busca aferir se os resultados 
obtidos pelo sistema condizem com a realidade. Para tal, manualmente contou-se os buracos em uma determinada trajetória dentro da via, e após percorreu-se por 8 vezes o mesmo percurso mapeado.

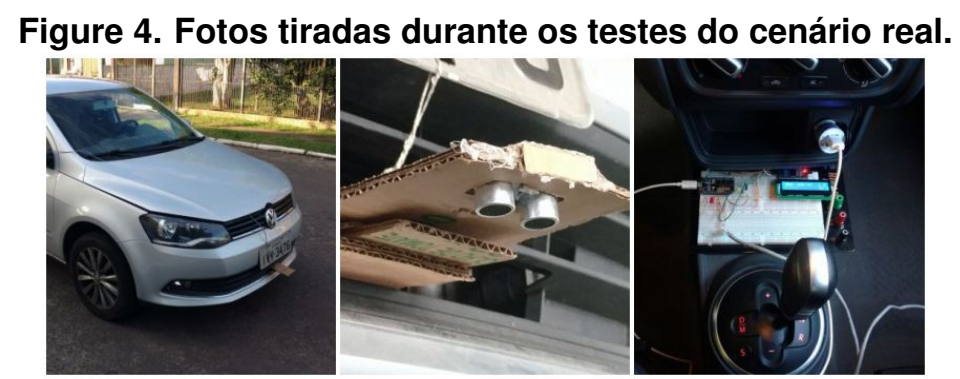

A Figura 4 mostra 3 fotos que exemplificam a instalação do sistema no veículo. A primeira mostrando a posição da instalação do sensor na parte frontal, a segunda mostrando o case criado e montado manualmente que envolve o sensor para apoia-lo e protege-lo, e a terceira a instalação da placa controladora no interior do veículo.

Figure 5. Buracos mapeados durante o teste em ambiente real.

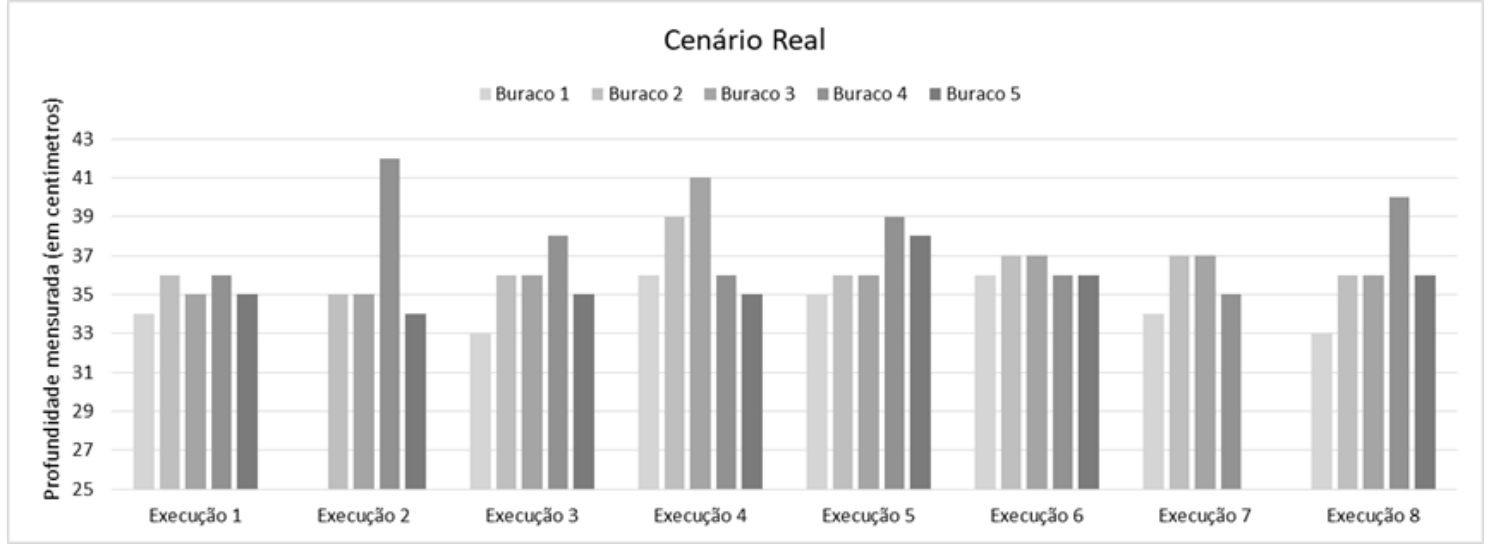

Durante a execução dos testes no ambiente real, o sistema apresentou um comportamento anormal, detectando buracos onde não havia. De forma a tentar calibrar o sistema, manipulou-se algumas variáveis de ajuste do algoritmo de análise. Dentre essas variáveis, o tempo de aguardo entre os ciclos de leitura do sensor e a tolerância dos valores lidos. Os testes demonstraram que não era uma questão de trabalhar com a tolerância, mas sim verificar a imprecisão das leituras fornecidas pelo sensor HC-SR04 que não se apresentava nos testes no cenário simulado. Neste quesito notou-se a deficiência deste sensor em efetuar leituras de determinadas superfícies não completamente sólidas, como é o caso da manta asfáltica usada na pavimentação das vias brasileiras.

O gráfico apresentado na Figura 5 apresenta as profundidades mensuradas (em centímetros) para cada um dos cinco buracos em cada uma das oito execuções dos teste. Evidencia-se que neste cenário nem todos os buracos foram mapeados (buraco $1 \mathrm{x}$ execução 2 e buraco 5 x execução 7), todavia, todos aqueles que foram detectados foram corretamente enviados ao aplicativo Android. Também é possível observar as oscilações que ocorrem entre as execuções para o mesmo buraco, corroborando com a indicação de que o sensor HC-SR04 não opera adequadamente em determinadas superfícies. 
Entretanto, a leitura por parte do sensor é apenas um ponto técnico e especifico relacionado a uma instancia dos add-ons, e seu mal funcionamento não implica na invalidação do modelo. O modelo mostra-se válido visto que a imperfeição na detecção de buracos deste add-on pode ser corrigida substituindo por outra tecnologia.

\subsection{Cenário: teste de notificação ao motorista pelo aplicativo}

Este cenário prático de teste visa demonstrar e aferir o funcionamento da funcionalidade de notificação na forma visual e sonora ao motorista durante o trajeto, dado a existência de um(s) buraco(s) à frente. Para tal, foram pré-inseridas no sistema incidências de buracos dispostos aleatoriamente ao longo de duas localidades no município de Gravataí/RS e percorreu-se três vezes cada trecho que continha os buracos. Com o aplicativo DETAB aberto durante a condução de um veículo trafegando a aproximadamente $40 \mathrm{~km} / \mathrm{h}$, observou-se que o aplicativo notificou corretamente todas as incidências de buracos, durante todas as seis execuções, indicando que o módulo de notificação do usuário está comunicando-se adequadamente com os demais e operando plenamente sua função.

Figure 6. Exemplo de telas do aplicativo Android.

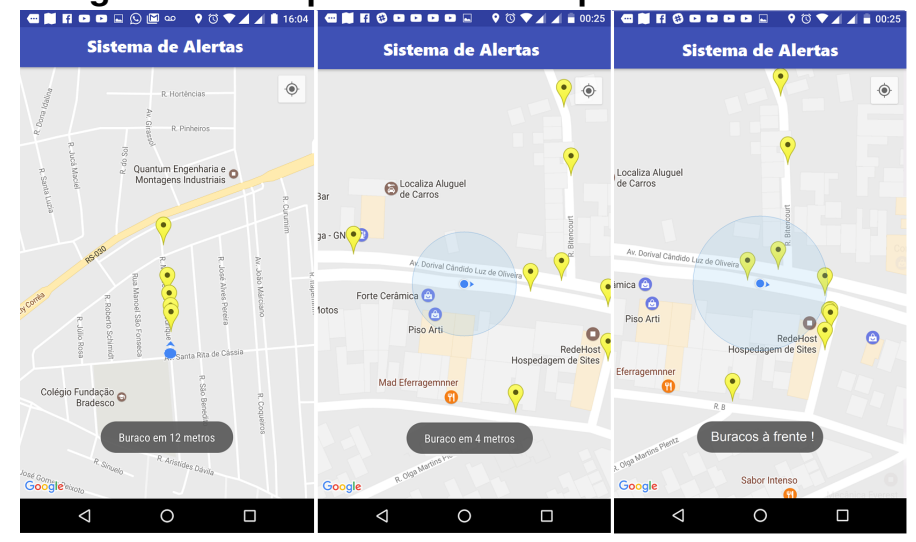

A Figura 6 mostra três screenshots da tela do smartphone durante a exibição de notificações sobre buracos à frente (sonora e visual), a onde o sistema foi previamente configurado para alertar o motorista quando a distância do veículo em relação ao buraco for igual ou inferior a 15 metros, mas este valor pode ser configurado com outros valores para haver um tempo hábil maior para tomada de decisões. Devido a precisão do sistema GPS não é possível afirmar exatamente em qual faixa da via o buraco está presente, sendo notificado inclusive buracos que não necessariamente estão na rota frontal do veículo.

\section{Conclusão e Trabalhos Futuros}

Este trabalho apresenta o DETAB, um modelo de detecção de buracos e alertas à motoristas sobre a incidência de buracos, prevenindo acidentes e fornecendo dados à órgãos que planejam e executam ações de reparo nem vias asfaltadas. Neste trabalho, priorizou-se o baixo custo, valendo-se de um sensor ultrassônico simples e um aplicativo para Android, presente na maior parte dos dispositivos moveis brasileiros [WorldPanel 2016].

Com base nos testes, constate-se evidências do funcionamento do DETAB e da capacidade de identificar buracos, ser colaborativo ao enviar informações coletadas para a API, e por distribuir essa informação a outros motoristas. Entretanto, considerando-se 
que os testes exitosos foram em sua maioria no ambiente simulado, é importante salientar que os resultados obtidos não podem ser generalizados para qualquer tipo de via.

Como trabalhos futuros, espera-se testar outros sensores que proporcionem maior precisão ao sistema. Além disto, a escolha de outros sensores e formas de mapear o solo permitirá antever os buracos até mesmo para o primeiro motorista a cursar um trajeto. Uma integração com o sistema On Board Diagnostics (ODB-II) dos veículos pode fornecer parâmetros úteis a serem usados para alertar o motorista viabilizando uma aproximação e manobra de desvio cada vez mais segura.

\section{References}

CNT, C. N. d. T. (2016). Anuário cnt do transporte - estatísticas consolidadas. Disponível em: http : //anuariodotransporte.cnt.org.br/File/PrincipaisDados.pdf. Acesso em: 09 Junho 2017.

CNT, C. N. d. T. (2018). O estudo transporte rodoviário: Rodovias esquecidas do brasil. Disponível em: http : //cms.cnt.org.br/ImagensCNT/PDFsCNT/Estudo RodoviasEsquecidas/rodovias - esquecidas - brasil.pdf. Acesso em: 30 Março 2018.

Klein, B. and Borges, C. E. L. (2016). Buracos monitor. Disponível em: http : //www.buracosmonitor.com.br/blog/2016/08/quem - somos/. Acesso em: 09 Junho 2017.

Madli, R., Hebbar, S., Pattar, P., and Golla, V. (2015). Automatic detection and notification of potholes and humps on roads to aid drivers. IEEE Sensors Journal, 15(8):43134318.

Moazzam, I., Kamal, K., Mathavan, S., Usman, S., and Rahman, M. (2013). Metrology and visualization of potholes using the microsoft kinect sensor. In 16th International IEEE Conference on Intelligent Transportation Systems (ITSC 2013), pages 1284-1291. IEEE, IEEE.

Murthy, S. B. S. and Varaprasad, G. (2014). Detection of potholes in autonomous vehicle. IET Intelligent Transport Systems, 8(6):543-549.

Nienaber, S., Kroon, R. S., and Booysen, M. J. (2015). A comparison of low-cost monocular vision techniques for pothole distance estimation. In Computational Intelligence, 2015 IEEE Symposium Series on, pages 419-426. IEEE.

Rishiwal, V. and Khan, H. (2016). Automatic pothole and speed breaker detection using android system. In 2016 39th International Convention on Information and Communication Technology, Electronics and Microelectronics (MIPRO), pages 1270-1273. IEEE.

Vupparaboina, K. K., Tamboli, R. R., Shenu, P. M., and Jana, S. (2015). Laser-based detection and depth estimation of dry and water-filled potholes: A geometric approach. In Communications (NCC), 2015 Twenty First National Conference on, pages 1-6. IEEE.

WorldPanel, K. (2016). Smartphone os market share. Disponível em: http : //www.kantarworldpanel.com/global/smartphone - os - market - share/. Acesso em: 09 Junho 2017. 